\title{
Time Scale Discrete Fourier Transforms
}

\author{
John M. Davis \\ Department of Mathematics \\ Baylor University \\ Waco, TX 76798 \\ Email: John_M_Davis@ baylor.edu
}

\author{
Ian A. Gravagne and Robert J. Marks II \\ Department of Electrical and Computer Engineering \\ Baylor University \\ Waco, TX 76798 \\ Email: Ian_Gravagne@baylor.edu, \\ Robert_Marks@baylor.edu
}

\begin{abstract}
The discrete and continuous Fourier transforms are applicable to discrete and continuous time signals respectively. Time scales allows generalization to to any closed set of points on the real line. Discrete and continuous time are special cases. Using the Hilger exponential from time scale calculus, the discrete Fourier transform (DFT) is extended to signals on a set of points with arbitrary spacing. A time scale $\mathbb{D}_{N}$ consisting of $N$ points in time is shown to impose a time scale (more appropriately dubbed a frequency scale), $\mathbb{U}_{N}$, in the Fourier domain The time scale DFT's (TS-DFT's) are shown to share familiar properties of the DFT, including the derivative theorem and the power theorem. Shifting on a time scale is accomplished through a boxminus and boxplus operators. The shifting allows formulation of time scale convolution and correlation which, as is the case with the DFT, correspond to multiplication in the frequency domain.
\end{abstract}

\section{INTRODUCTION}

A time scale is any collection of closed points on the real line. Continuous time, $\mathbb{R}$, and discrete time $\mathbb{Z}$, are special cases. The calculus of time scales was introduced by Hilger [11]. Time scales have found utility in describing the behavior of dynamic systems [1], [13] and have been applied to control theory [3], [4], [5], [7], [10].

On $\mathbb{R}$ and $\mathbb{Z}$, respectively, the continuous time and discrete time Fourier transforms are well studied [16]. Properties of the Laplace and Fourier transforms on time scales have been extended to time scales with unbounded domains [1], [6], [8], [9], [12], [14], [16].

The conventional discrete Fourier transform (DFT) is defined over a finite number of uniformly spaced points. This paper extends the DFT to a finite number of discrete time points that are not uniformly spaced. ${ }^{1}$ The time scale of a finite number of $N$ discrete points, $\mathbb{D}_{N}$, is shown to uniquely map into a frequency scale (a time scale in the frequency domain), $\mathbb{U}_{N}$, in the Fourier domain. Familiar Fourier transform theorems, including the shift, convolution and derivative theorems, are shown to generalize to the time scale DFT (TS-DFT).

\section{TIME SCALES}

Our introduction to time scales is limited to that needed to establish notation. A more detailed explanation are in our previous papers [4], [5], [6], [8], [9], [10], [13], [14], [16] and

\footnotetext{
1 Our development is distinct from the time scale Fourier transform proposed by Hilger [12], [14], [16]. Our treatment more closely resembles Laplace transform generalizations where two signals on a time scale $\mathbb{T}$, when convolved, result in a signal on the same time scale, $\mathbb{T}[1],[6],[8],[9]$.
}

a complete rigorous treatment is in the text by Bohner and Peterson [1].

1) A time scale, $\mathbb{T}$, is any collection of closed intervals on the real line. Generally, the time scale can contain both discrete time points and continuous time intervals. Since our development of TS-DFT is only on time scales containing discrete points, we henceforth restrict attention to time scales containing discrete points, ${ }^{2}$ denoted $\mathbb{D}$. Discrete time, $\mathbb{Z}$, is a special case.

2) The graininess, $\mu(t)$, is the distance between adjacent points in a time scale at time $t \in \mathbb{T}$ and is defined generally by

$$
\mu(t)=\left(\inf _{\tau>t, \tau \in \mathbb{T}} \tau\right)-t .
$$

For $\mathbb{D}$,

$$
\mu\left(t_{n}\right)=t_{n+1}-t_{n} .
$$

3) The Hilger derivative of a function $x(t)$ at $t \in \mathbb{T}$ is

$$
x^{\Delta}(t):=\frac{x(t+\mu(t))-x(t)}{\mu(t)} .
$$

When $\mu(t)=d t \quad(=0)$, the Hilger derivative is interpreted in the limiting sense and

$$
x^{\Delta}(t)=\frac{d}{d t} x(t)
$$

For $\mathbb{D}$, we have

$$
x^{\Delta}\left(t_{n}\right)=\frac{x\left(t_{n+1}\right)-x\left(t_{n}\right)}{\mu\left(t_{n}\right)} .
$$

4) If $y(t)=x^{\Delta}(t)$, then the definite time scale integral is

$$
\int_{a}^{b} y(t) \Delta t=x(b)-x(a) .
$$

For $\mathbb{D}$, we have [1]

$$
\int_{t_{p}}^{t_{q}} y(t) \Delta t=\sum_{n=p}^{q-1} y\left(t_{n}\right) \mu\left(t_{n}\right) .
$$

5) When $x(0)=1$, the solution to the Hilger differential equation,

$$
x^{\Delta}(t)=z x(t)
$$

${ }^{2}$ We use $\mathbb{D}$ to denote a time scale with an arbitrary, possibly infinite, set of discrete isolated points. The notation $\mathbb{D}_{N}$ indicates the time scale has $N$ points. 
is $x(t)=e_{z}(t)$ where the generalized exponential is

$$
e_{z}(t):=\exp \left(\int_{\tau=0}^{t} \frac{\ln (1+z \mu(\tau))}{\mu(\tau)} \Delta \tau\right) .
$$

For $\mathbb{D}$ and $n>0$,

$$
e_{z}\left(t_{n}\right)=\prod_{m=0}^{n-1}\left(1+z \mu\left(t_{m}\right)\right)
$$

Since $\mu\left(t_{m}\right)$ is real,

$$
e_{z}^{*}\left(t_{n}\right)=e_{z^{*}}\left(t_{n}\right)
$$

The properties of the generalized exponential parallel those of $z^{n}$ for the $z$-transform and $e^{j \omega t}$ for the Fourier transform are responsible for the utility of the TS-DFT.

\section{Time Scale Exponential Basis Sets}

Consider a time scale, $\mathbb{D}_{N}$, of $N+1$ real temporal points, $\left\{t_{n} \mid 0 \leq n \leq N\right\}$ with $t_{0}=0 \leq t_{n}<t_{n+1} \leq t_{N}$. (The point $t_{N}$ is required to determine the graininess of the point $t_{N-1}$.) Let $x\left(t_{n}\right)$ and $h\left(t_{n}\right)$ be images on $\mathbb{D}_{N}$. Define the inner product

$$
\left\langle x\left(t_{n}\right) \mid h\left(t_{n}\right)\right\rangle=\sum_{n=0}^{N-1} x\left(t_{n}\right) h^{*}\left(t_{n}\right) w\left(t_{n}\right)
$$

where $w\left(t_{n}\right)>0$ is a weighting function and the asterisk denotes complex conjugation. Generally, $w\left(t_{n}\right)$ is arbitrary but, to make the integration constant with time scale integration, we will henceforth use the graininess as the weight, i.e. $w\left(t_{n}\right)=\mu\left(t_{n}\right)$ The corresponding norm is

$$
\|x(t)\|=\sqrt{\left\langle x\left(t_{n}\right) \mid x\left(t_{n}\right)\right\rangle} .
$$

Two time scale exponentials are orthogonal if

$$
\left\langle e_{z}\left(t_{n}\right) \mid e_{\varsigma}\left(t_{n}\right)\right\rangle=0 \text { for } z \neq \varsigma .
$$

If we set $\varsigma=0$, then applying (1) and (3) to (4) gives

$$
\begin{aligned}
& \left.\left\langle e_{z}\left(t_{n}\right) \mid e_{\varsigma}\left(t_{n}\right)\right\rangle\right|_{\varsigma=0}=\left\langle e_{z}\left(t_{n}\right) \mid 1\right\rangle \\
& =\sum_{n=0}^{N-1} e_{z}\left(t_{n}\right) \mu\left(t_{n}\right) \\
& =\mu\left(t_{0}\right)+\sum_{n=1}^{N-1} \mu\left(t_{n}\right) \prod_{m=0}^{n-1}\left(1+z \mu\left(t_{m}\right)\right) \\
& =0 \text { for } z \neq \varsigma=0 .
\end{aligned}
$$

The solution of the $N-1$ st order polynomial can be used to generate orthogonal time scale exponentials. Motivated by (5), we dub the roots of the polynomial

$$
\mu\left(t_{0}\right)+\sum_{n=1}^{N-1} \mu\left(t_{n}\right) \prod_{m=0}^{n-1}\left(1+z \mu\left(t_{m}\right)\right)=0
$$

the frequency roots of the time scales, $\mathbb{D}_{N}$.

\section{A. Example Frequency Roots}

Here are some examples of frequency roots from the polynomial in (6).
1) On a Time Scale of Uniformly Spaced Points: The time scale of uniformly spaced points is the time scale conventionally associated with the DFT. Then $\mu\left(t_{n}\right)=1$ and (5) becomes

$$
\begin{aligned}
\left\langle e_{z}\left(t_{n}\right) \mid 1\right\rangle & =1+\sum_{n=1}^{N-1} \prod_{m=0}^{n-1}(1+z) \\
& =\sum_{n=0}^{N-1}(1+z)^{n}=0 .
\end{aligned}
$$

This is a geometric series with solution

$$
\left\langle e_{z}\left(t_{n}^{\sigma}\right) \mid 1\right\rangle=\frac{(1+z)^{N}-1}{z}=0 .
$$

Note that the zeroth order term in the numerator is zero, so since $z \neq \varsigma=0,(7)$ is an $(N-1)$ st polynomial with $N-1$ frequency roots. For $z \neq \varsigma=0$ (required by (4)) and $z \neq-1$ (the regressivity condition for $\mathbb{Z}[1]$ ), this equation is satisfied when $(1+z)^{N}=\exp (-j 2 \pi k)$ where $k$ is an arbitrary integer. Thus the $N-1$ polynomial frequency roots are

$$
z_{k}=-1+e^{-j 2 \pi k / N} ; 0<k<N \text {. }
$$

As shown in Figure 1, these are points equally spaced on a unit circle centered at $z=-1$.

Other time scales do not lend themselves to the ease of analysis afforded by the time scale of uniformly spaced points.

2)On a log time scale we have $\mathbb{D}_{N}=\left\{t_{n}=\log _{2}(n)\right\}$. Example frequency roots of this time scale are shown in Figure 1.

3)The Harmonic Time Scale is defined as

$$
t_{n}=\left\{\begin{array}{ccc}
0 & ; & n=0 \\
\sum_{k=1}^{n} \frac{1}{k} & ; & n>0 .
\end{array}\right.
$$

The frequency roots are shown in Figure 1 for $N=16$.

4)The Geometric Time Scale for a parameter $q>0$, is defined as ${ }^{3}$

$$
t_{n}=\left\{\begin{array}{ccc}
0 & ; & n=0 \\
q^{n} & ; & n>0
\end{array}\right.
$$

The frequency roots are shown in Figure 2 for $N=16$.

For $q<1$, the values of the time scale are the same as in (9) except they are arranged in ascending order.

5)The Poisson Time Scale chooses points in a Poisson process [16] with parameter $\lambda$. The origin, $t_{0}=0$, is then included. Example frequency roots are shown in Figure 3.

\section{B. Basis Examples}

Here are some examples of time scale exponential basis sets for some example time scales. For the exponential basis plots in Figure $4, N=8$. Points are linearly connected. The location of points on the time scale are marked along the time axis with dots.

1) On a Time Scale with Uniformly Spaced Points the TSDFT becomes the conventional DFT [16]. For the uniformly

\footnotetext{
${ }^{3}$ This differs from the quantum time scale [2] which includes the origin and points $q^{n}$ for $n \in \mathbb{Z}$.
} 

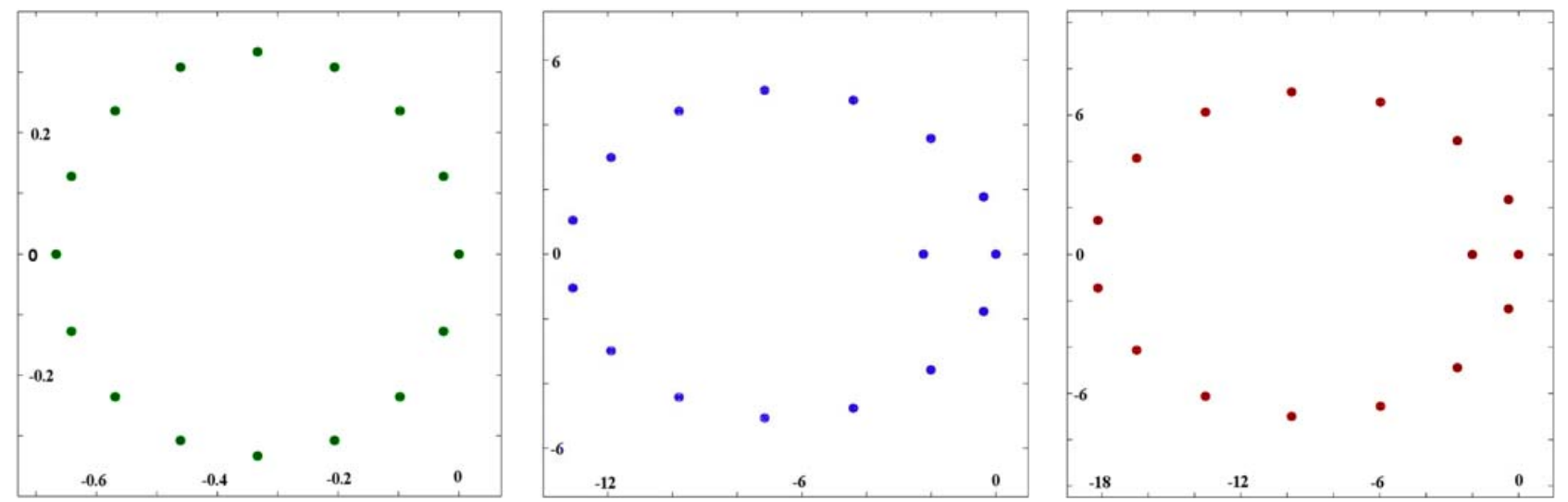

Fig. 1. Frequency roots of some time scales. In each plot, the horizontal and vertical scales are the same. LEFT: The frequency roots of the time scales with equally spaced points lie on a shifted circle in the $z$ plane, dubbed the Hilger circle [1]. MIDDLE: The frequency roots of the log time scale, plotted on the complex $z$ plane, discussed in Section III-A1 for $N=16$. RIGHT: The frequency roots of the harmonic scale, plotted on the complex $z$ plane, as discussed in Section III-A1 for $N=16$.
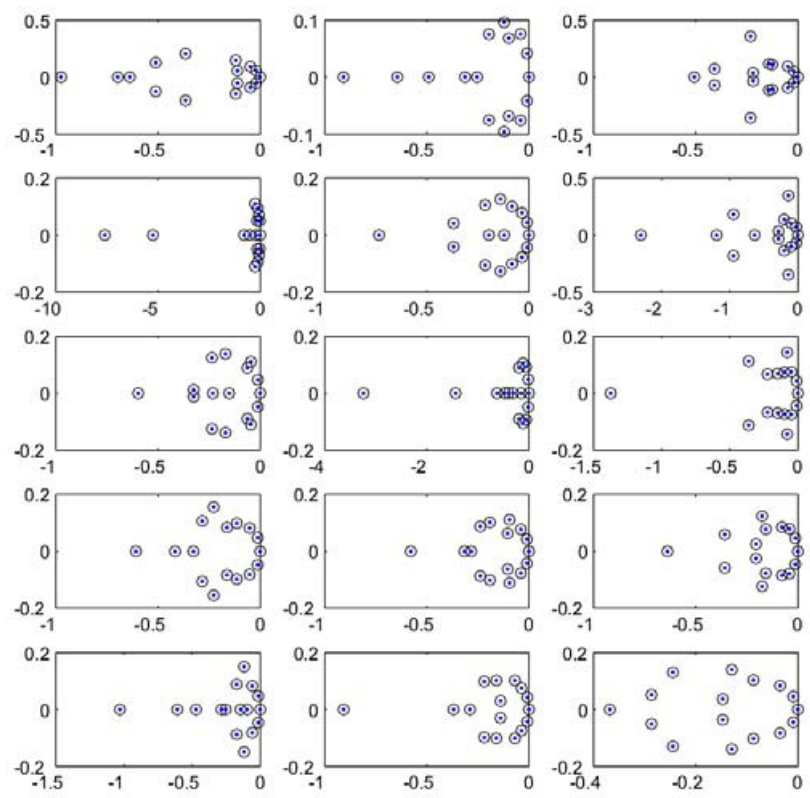

Fig. 3. The frequency roots of 15 realizations of a Poisson time scale with parameter $\lambda=1$ point per interval. The shape of the root locations varies considerably. Note that scales can differ from plot to plot.

spaced points described in (III-A1), the orthonormalized exponential basis functions are

$$
e_{z_{k}}\left(t_{n}\right)=e^{j 2 \pi n k / N} ; \text { for } 0 \leq n, k<N .
$$

Proof: Since $\mu\left(t_{n}\right)=1$, we have from (1)

$$
e_{z_{k}}\left(t_{n}\right)=\prod_{m=0}^{n-1}(1+z)=(1+z)^{n} .
$$

Substituting (8) gives (9). These are the familiar normalized basis functions for the discrete Fourier transform (DFT) and are shown in Figure 4.
1) Other Basis Sets: The orthonormalized basis functions for the log time scale, the harmonic time scale, and the geometric time scale for $q=1.2$ are shown in Figure 4 .

\section{Orthogonal Expansions and Inversion}

When the orthogonal basis $\left\{e_{z_{k}}\left(t_{n}\right) \mid 0 \leq n, k<N\right\}$ is complete, we can expand any function, $x\left(t_{n}\right)$, on the time scale as

$$
x\left(t_{n}\right)=\sum_{\ell=0}^{N-1} c_{\ell} e_{z_{\ell}}\left(t_{n}\right)
$$

where $c_{n}$ are the series expansion coefficients. Let

$$
\begin{aligned}
X\left(z_{k}\right) & :=\sum_{n=0}^{N-1} x\left(t_{n}\right) e_{z_{k}}^{*}\left(t_{n}\right) \mu\left(t_{n}\right) \\
& =\sum_{n=0}^{N-1}\left[\sum_{\ell=0}^{N-1} c_{\ell} e_{z_{\ell}}\left(t_{n}\right)\right] e_{z_{k}}^{*}\left(t_{n}\right) \mu\left(t_{n}\right) \\
& =\sum_{\ell=0}^{N-1} c_{\ell}\left[\sum_{n=0}^{N-1} e_{z_{\ell}}\left(t_{n}\right) e_{z_{k}}^{*}\left(t_{n}\right) \mu\left(t_{n}\right)\right] \\
& =\sum_{\ell=0}^{N-1} c_{\ell}\left[\left\|e_{z_{k}}\right\|^{2} \delta[\ell-k]\right] \\
& =c_{k}\left\|e_{z_{k}}\right\|^{2} .
\end{aligned}
$$

Thus

$$
c_{k}=\frac{X\left(z_{k}\right)}{\left\|e_{z_{k}}\right\|^{2}}
$$

where

$$
\left\|e_{z_{k}}\right\|^{2}:=\sum_{n=0}^{N-1}\left|e_{z_{k}}\left(t_{n}\right)\right|^{2} \mu\left(t_{n}\right) .
$$




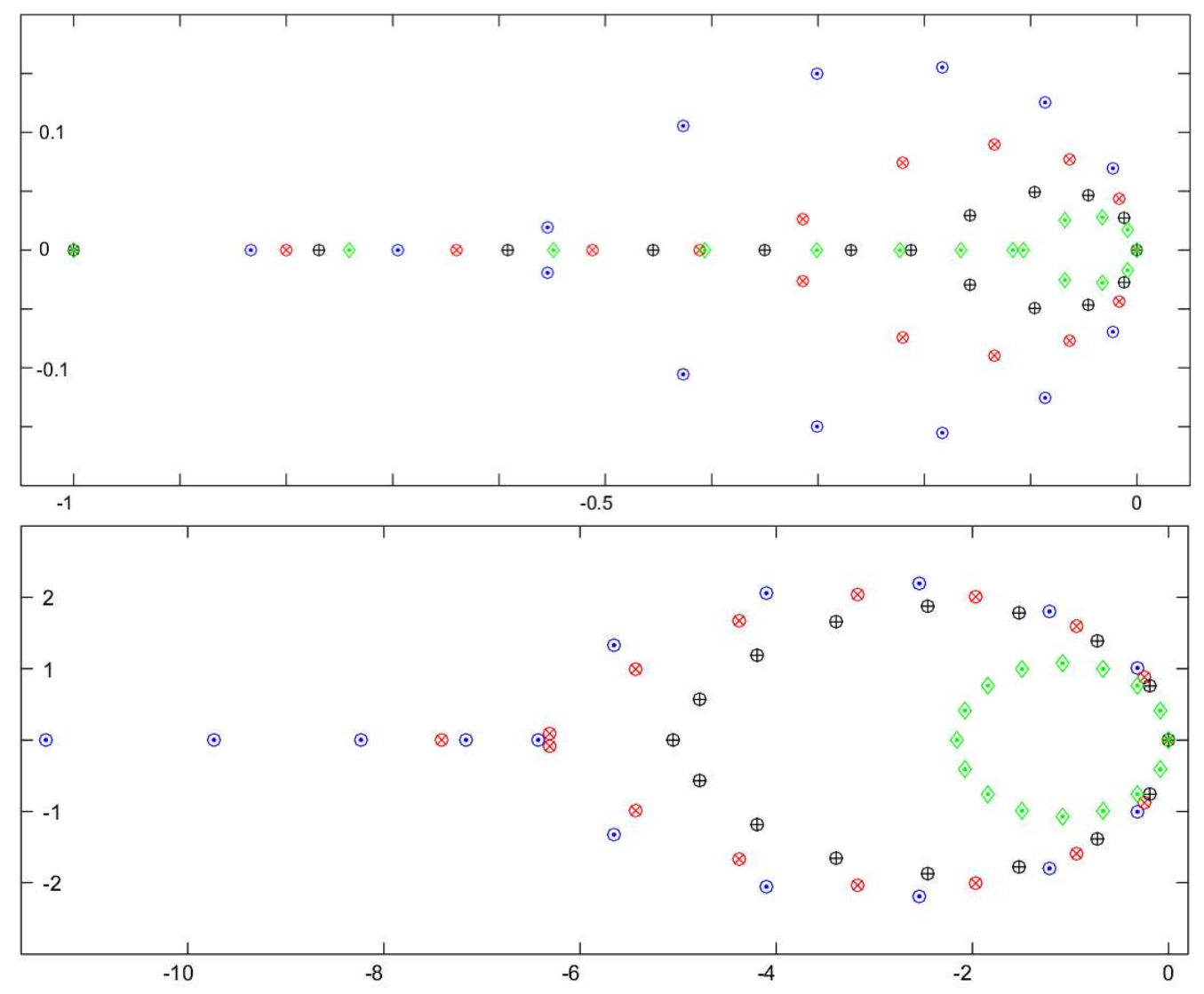

Fig. 2. TOP: The frequency roots of the geometric time scale in (9), plotted on the complex $z$ plane, as discussed in Section III-A1 for $N=16$. The values of $q$ are $1.20 \odot, 1.25 \otimes, 1.30 \oplus$, and $1.35 \diamond$. BOTTOM: The frequency roots of the geometric time scale, plotted on the complex $z$ plane, as discussed in Section III-A1 for $N=16$. The values of $q$ are $0.85 \odot, 0.875 \otimes, 0.90 \oplus$, and $0.99 \diamond$.

We find the following notation useful. ${ }^{4}$

$$
\partial t_{n}:=\mu\left(t_{n}\right)
$$

and

$$
\partial z_{k}:=\frac{1}{\left\|e_{z_{k}}\right\|^{2}} .
$$

Note that if $t_{n}$ has units of time, then $\partial t_{n}$ has units of time and $\partial z_{k}$ has units of reciprocal time.

From this analysis, we define the time scale DFT (TS-DFT) and its inverse.

$$
\begin{gathered}
\text { \TS-DFT } \\
x\left(t_{n}\right) \leftrightarrow X\left(z_{k}\right)=\sum_{n=0}^{N-1} x\left(t_{n}\right) e_{z_{k}}^{*}\left(t_{n}\right) \partial t_{n} . \\
\qquad \text { Inverse TS-DFT } \\
x\left(t_{n}\right)=\sum_{k=0}^{N-1} X\left(z_{k}\right) e_{z_{k}}\left(t_{n}\right) \partial z_{k} \leftrightarrow X\left(z_{k}\right) .
\end{gathered}
$$

\footnotetext{
${ }^{4}$ An alternate possibly more representative notation might be $\mu_{\mathbb{D}}\left(t_{n}\right)$ in lieu of $\partial t_{n}$ and $\mu_{\mathbb{U}}\left(z_{k}\right)$ instead of $\partial z_{k}$. We have opted for the shorter more compact notation.
}

Thus $x\left(t_{n}\right)$ is a finite duration signal on a time scale $\mathbb{D}_{N}$ with graininess $\partial t_{n}=\mu\left(t_{n}\right)$. This imposes a frequency scale, $\mathbb{U}_{N}$, with values $X\left(z_{k}\right)$ and graininess $\partial z_{k}$ given by (10). Thus

$$
u_{k}= \begin{cases}0 & ; k=0 \\ \sum_{\ell=1}^{k} \partial z_{\ell}=z_{k-1}+\partial z_{k} & ; \quad 1 \leq k \leq N .\end{cases}
$$

define the point locations on the time scale $\mathbb{U}_{N}$. The image $X\left(z_{k}\right)$ is assigned to the point ${ }^{5} u_{k}$.

- Conjugate Symmetry. When $x\left(t_{n}\right)$ is real, $X^{*}\left(z_{k}\right)=X\left(z_{k}^{*}\right)$. Proof: The proof results immediately upon applying (2) to the TS-DFT definition in (11).

\section{TS-DFT Transform Theorems}

Here are some theorems that parallel the conventional DFT [16].

- Area Theorem. Since $t_{0}=0$,

$$
X\left(z_{0}\right)=\sum_{n=0}^{N-1} x\left(t_{n}\right) \partial t_{n}
$$

5 An alternate notation might be $X\left(u_{k}\right)$ in lieu of $X\left(z_{k}\right)$. We choose to continue with the notation $X\left(z_{k}\right)$. 

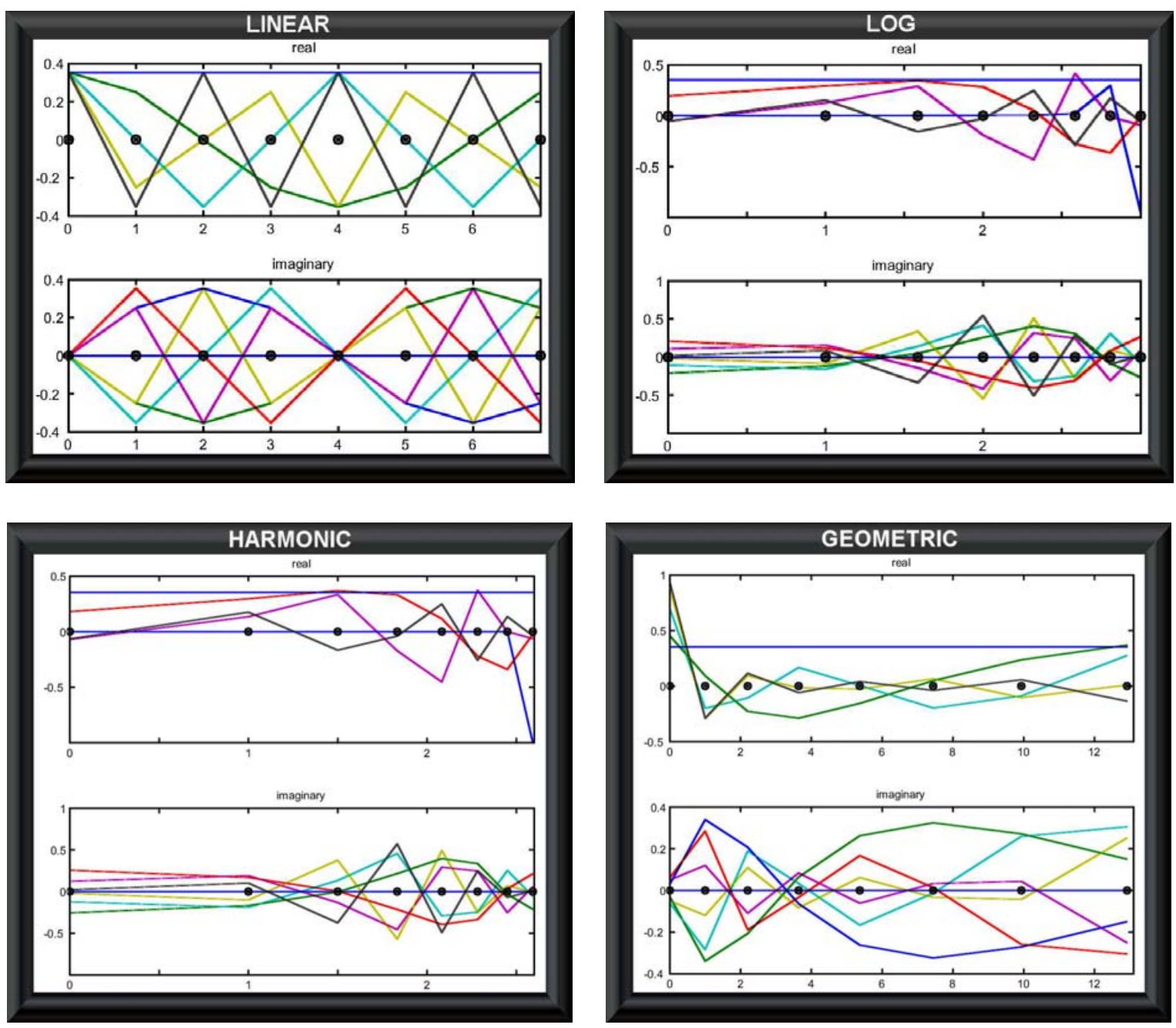

Fig. 4. The real (top) and imaginary (bottom) components of the orthonormalized basis functions for the linear time scale using $N=8$. The LINEAR plots are the familiar sins and cosines of the DFT kernel. The GEOMETRIC time scale is for $q=1.2$

Proof: Since $z_{0}=0$ and $e_{z}(0)=1$, this follows immediately from the TS-DFT definition in (11). Likewise,

$$
x\left(t_{0}\right)=\sum_{k=0}^{N-1} X\left(z_{k}\right) \partial z_{k}
$$

- Conjugate Symmetry. When $x\left(t_{n}\right)$ is real, $X^{*}\left(z_{k}\right)=X\left(z_{k}^{*}\right)$. Proof: The proof results immediately upon applying (2) to the TS-DFT definition in (11).

- Power Theorem.

$$
\sum_{n=0}^{N-1} x\left(t_{n}\right) h^{*}\left(t_{n}\right) \partial t_{n}=\sum_{k=0}^{N-1} X\left(z_{k}\right) H^{*}\left(z_{k}\right) \partial z_{k} .
$$

Proof: Follows from the definition of the TS-DFT in (11) and its inverse in (12)

$$
\begin{aligned}
& \sum_{k=0}^{N-1} X\left(z_{k}\right) H^{*}\left(z_{k}\right) \partial z_{k} \\
& =\sum_{k=0}^{N-1} X\left(z_{k}\right)\left[\sum_{n=0}^{N-1} h\left(t_{n}\right) e_{z_{k}}^{*}\left(t_{n}\right) \partial t_{n}\right]^{*} \partial z_{k} \\
& =\sum_{n=0}^{N-1}\left[\sum_{k=0}^{N-1} X\left(z_{k}\right) e_{z_{k}}\left(t_{n}\right) \partial z_{k}\right] h^{*}\left(t_{n}\right) \partial t_{n} \\
& =\sum_{n=0}^{N-1} x\left(t_{n}\right) h^{*}\left(t_{n}\right) \partial t_{n}
\end{aligned}
$$

Parseval's Theorem is a special case of the power theorem when $x=h$.

$$
\sum_{n=0}^{N-1}\left\|x\left(t_{n}\right)\right\|^{2} \partial t_{n}=\sum_{k=0}^{N-1}\left\|X\left(z_{k}\right)\right\|^{2} \partial z_{k}
$$

- Derivative theorem.

$$
x^{\Delta}\left(t_{n}\right) \leftrightarrow z_{k} X\left(z_{k}\right),
$$



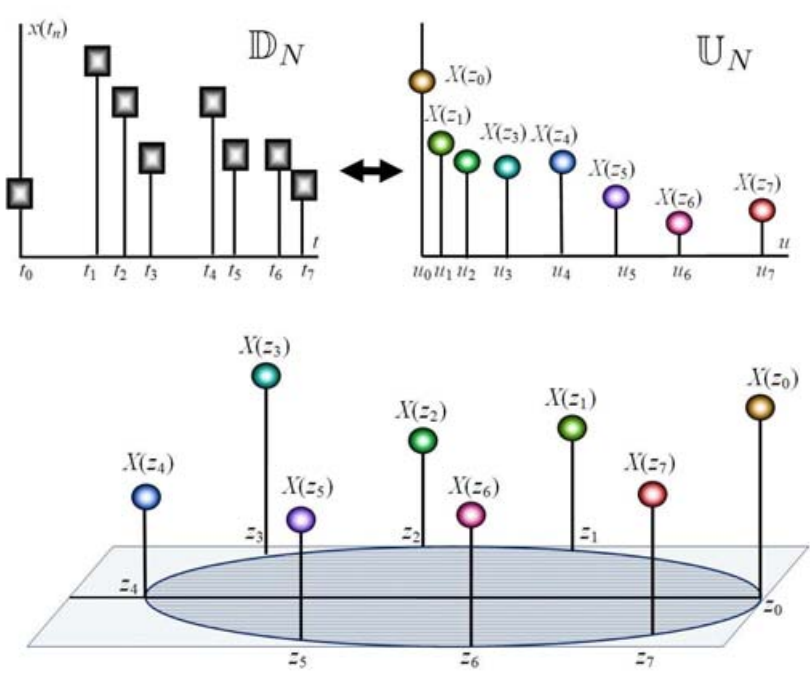

Fig. 5. A graphical illustration of the TS-DFT. On the upper left is a signal, $x\left(t_{n}\right)$, on a time scale $\mathbb{D}_{N}$ where, here, $N=8$. The time scale $\mathbb{D}_{N}$ dictates the frequency roots, $z_{k}$, as illustrated in Figures 1, 2 and 3 and the exponential basis sets illustrated in Figure 4 . The basis set applied to the signal $x\left(t_{n}\right)$ gives the values of the TS-DFT, namely $X\left(z_{k}\right)$, as illustrated in the bottom figure. The norms of the basis set components determines the $\partial z_{k}$ 's in (10) which, in turn, determines the frequency scale, $\mathbb{U}_{N}$, in (13). This is shown in the upper right.

Proof: From (12),

$$
\begin{aligned}
x^{\Delta}\left(t_{n}\right) & =\sum_{k=0}^{N-1} X\left(z_{k}\right) e_{z_{k}}^{\Delta}\left(t_{n}\right) \partial z_{k} \\
& =\sum_{k=0}^{N-1}\left[z_{k} X\left(z_{k}\right)\right] e_{z_{k}}\left(t_{n}\right) \partial z_{k},
\end{aligned}
$$

from which (15) follows.

\section{Shifts on a Time Scale}

The boxminus shift operator, $\boxminus$, on an arbitrary function, $h\left(t_{n}\right)$ on $\mathbb{D}_{N}$, is defined by its TS-DFT.

$$
h\left(t_{n} \boxminus t_{m}\right) \leftrightarrow H\left(z_{k}\right) e_{z_{k}}^{*}\left(t_{m}\right) .
$$

- The Hilger delta [6] is defined as

$$
\delta\left(t_{n}\right):=\frac{\delta[n]}{\partial t_{0}}
$$

where $\delta[n]$ is the Kronecker delta ${ }^{6}$ and we have used $t_{0}=0$.

- The TS-DFT of the Hilger delta is

$$
\delta\left(t_{n}\right) \leftrightarrow 1
$$

Proof: The proof follows directly from the TS-DFT in (11).

- The shifted Hilger delta and its TS-DFT is

\begin{tabular}{|c|c|c|c|}
\hline TS-DFT & $x\left(t_{n}\right)$ on $\mathbb{D}_{N}$ & $\leftrightarrow$ & $X\left(z_{k}\right)$ on $\mathbb{U}_{N}$ \\
\hline transform & $X\left(z_{k}\right)$ & $=$ & $\sum x\left(t_{n}\right) e_{z_{k}}^{*}\left(t_{n}\right) \partial t_{n}$ \\
\hline inverse & $x\left(t_{n}\right)$ & $=$ & $\sum X\left(z_{k}\right) e_{z_{k}}\left(t_{n}\right) \partial z_{k}$ \\
\hline $\begin{array}{l}\text { area } \\
\text { theorem }\end{array}$ & $\sum x\left(t_{n}\right) \partial t_{n}$ & $=$ & $X\left(z_{0}\right)$ \\
\hline $\begin{array}{l}\text { symmetry } \\
(x \text { real })\end{array}$ & $X^{*}\left(z_{k}\right)$ & $=$ & $X\left(z_{k}^{*}\right)$ \\
\hline $\begin{array}{l}\text { power } \\
\text { theorem }\end{array}$ & $\sum x\left(t_{n}\right) h^{*}\left(t_{n}\right) \partial t_{n}$ & $=$ & $\sum X\left(z_{k}\right) H^{*}\left(z_{k}\right) \partial z_{k}$ \\
\hline $\begin{array}{l}\text { Parseval's } \\
\text { theorem }\end{array}$ & $\sum\left\|x\left(t_{n}\right)\right\|^{2} \partial t_{n}$ & $=$ & $\sum\left\|X\left(z_{k}\right)\right\|^{2} \partial z_{k}$ \\
\hline $\begin{array}{l}\text { box minus } \\
\text { shift theorem }\end{array}$ & $h\left(t_{n} \boxminus t_{m}\right)$ & $\leftrightarrow$ & $H\left(z_{k}\right) e_{z_{k}}^{*}\left(t_{m}\right)$ \\
\hline $\begin{array}{l}\text { inverted } \boxminus \\
\text { shift theorem }\end{array}$ & $x^{*}\left(t_{m} \boxminus t_{n}\right)$ & $\leftrightarrow$ & $X^{*}\left(z_{k}\right) e_{z_{k}}^{*}\left(t_{m}\right)$ \\
\hline $\begin{array}{l}\text { box minus } \\
\text { theorem }\end{array}$ & $x^{*}\left(\boxminus t_{n}\right)$ & $\leftrightarrow$ & $X^{*}\left(z_{k}\right)$ \\
\hline $\begin{array}{l}\text { box plus } \\
\text { shift theorem }\end{array}$ & $h\left(t_{n} \boxplus t_{m}\right)$ & $\leftrightarrow$ & $H\left(z_{k}\right) e_{z_{k}}\left(t_{m}\right)$ \\
\hline convolution $^{a}$ & $x\left(t_{n}\right) * h\left(t_{n}\right)$ & $\leftrightarrow$ & $X\left(z_{k}\right) H\left(z_{k}\right)$ \\
\hline correlation $^{b}$ & $x\left(t_{n}\right) \star h\left(t_{n}\right)$ & $\leftrightarrow$ & $X^{*}\left(z_{k}\right) H\left(z_{k}\right)$ \\
\hline derivative & $x^{\Delta}\left(t_{n}\right)$ & $\leftrightarrow$ & $z_{k} X\left(z_{k}\right)$ \\
\hline $\begin{array}{l}\text { frequency } \\
\text { response }\end{array}$ & $h\left(t_{n}\right) * e_{z_{k}}\left(t_{n}\right)$ & $=$ & $H\left(z_{k}\right) e_{z_{k}}\left(t_{n}\right)$ \\
\hline
\end{tabular}

$$
\delta\left(t_{n} \boxminus t_{m}\right)=\frac{\delta[n-m]}{\partial t_{m}} \leftrightarrow e_{z_{k}}^{*}\left(t_{m}\right) .
$$

Proof: Follows from application of (16) to (17).

$$
{ }^{6} \delta[n]=1 \text { for } n=0 \text { and is otherwise zero. }
$$

TABLE I

SOME TS-DFT THEOREMS. All SUMS ARE FROM 0 TO $N-1$, i.e OVER $n$ WE HAVE $\sum=\sum_{n=0}^{N-1}$ AND, OVER $k, \sum=\sum_{k=0}^{N-1}$. (A) CONVOLUTION IS DEFINED IN (28) AND (B) CORRELATION IN ( 30 ).

The sifting property of Hilger delta follows immediately as

$$
\sum_{m=0}^{N-1} x\left(t_{m}\right) \delta\left(t_{n} \boxminus t_{m}\right) \partial t_{m}=x\left(t_{n}\right) .
$$

Basis Exponential TS-DFT. The TS-DFT of a basis exponential is

$$
e_{z_{\ell}}\left(t_{n}\right) \leftrightarrow \delta\left(z_{k} \boxminus z_{\ell}\right)
$$

where

$$
\delta\left(z_{k} \boxminus z_{\ell}\right)=\frac{\delta[k-\ell]}{\partial z_{\ell}}
$$

is the Hilger delta on the time scale $\mathbb{D}$.

A special case is for $t_{n}=t_{0}=0$.

$$
1 \leftrightarrow \delta\left(z_{k}\right)
$$

Proof: Substitute (20) into (11) and use the orthogonal property in (4). 


\begin{tabular}{|c|c|c|c|}
\hline DC Values & $\begin{array}{l}e_{z_{0}}\left(t_{n}\right) \\
e_{z_{k}}\left(t_{0}\right)\end{array}$ & $\begin{array}{l}= \\
=\end{array}$ & $\begin{array}{l}1 \\
1\end{array}$ \\
\hline Hilger delta & $\delta\left(t_{n}\right):=\delta[n] / \partial t_{0}$ & $\leftrightarrow$ & 1 \\
\hline shifted Hilger delta & $\delta\left(t_{n} \boxminus t_{m}\right)$ & $\leftrightarrow$ & $e_{z_{k}}^{*}\left(t_{m}\right)$ \\
\hline \multirow[t]{2}{*}{ sifting property } & $\sum_{m=0}^{N-1} x\left(t_{m}\right)$ & & \\
\hline & $\times \delta\left(t_{n} \boxminus t_{m}\right) \mu\left(t_{m}\right)$ & $=$ & $x\left(t_{n}\right)$ \\
\hline convolution identity & $x\left(t_{n}\right) * \delta\left(t_{n}\right)$ & $=$ & $x\left(t_{n}\right)$ \\
\hline one & 1 & $\leftrightarrow$ & $\delta\left(z_{k}\right)=\delta[k] / \partial z_{\ell}$ \\
\hline basis exponential & $e_{z_{\ell}}\left(t_{n}\right)$ & $\leftrightarrow$ & $\delta\left(z_{k} \boxminus z_{\ell}\right)$ \\
\hline conjugate symmetry & $e_{z_{k}}^{*}\left(t_{n}\right)$ & $=$ & $e_{z_{k}^{*}}\left(t_{n}\right)$ \\
\hline \multirow[t]{3}{*}{ box minus shift } & $e_{z_{k}}\left(t_{n} \boxminus t_{m}\right)$ & $=$ & $e_{z_{k}}\left(t_{n}\right) e_{z_{k}}^{*}\left(t_{m}\right)$ \\
\hline & $e_{z_{k}}\left(\boxminus t_{n}\right)$ & $=$ & $e_{z_{k}}^{*}\left(t_{n}\right)$ \\
\hline & $e_{z_{k}}\left(t_{n} \boxminus t_{n}\right)$ & $=$ & $\left|e_{z_{k}}\left(t_{n}\right)\right|^{2}$ \\
\hline \multirow[t]{3}{*}{ box plus shift } & $e_{z_{k}}\left(t_{n} \boxplus t_{m}\right)$ & $=$ & $e_{z_{k}}\left(t_{n}\right) e_{z_{k}}\left(t_{m}\right)$ \\
\hline & $e_{z_{k}}\left(\boxplus t_{n}\right)$ & $=$ & $e_{z_{k}}\left(t_{n}\right)$ \\
\hline & $e_{z_{k}}\left(t_{n} \boxplus t_{n}\right)$ & $=$ & $\left(e_{z_{k}}\left(t_{n}\right)\right)^{2}$ \\
\hline
\end{tabular}

TABLE II

Properties of exponentials and Hilger deltas.

- Basis Exponential Shift. The box minus basis exponential shift can be written as

$$
e_{z_{\ell}}\left(t_{n} \boxminus t_{m}\right)=e_{z_{\ell}}\left(t_{n}\right) e_{z_{\ell}}^{*}\left(t_{m}\right)
$$

Proof: Applying (16) to (20) gives

$$
\begin{aligned}
e_{z_{\ell}}\left(t_{n} \boxminus t_{m}\right) & \leftrightarrow e_{z_{k}}^{*}\left(t_{m}\right) \delta\left(z_{k} \boxminus z_{\ell}\right) \\
& =e_{z_{\ell}}^{*}\left(t_{m}\right) \delta\left(z_{k} \boxminus z_{\ell}\right)
\end{aligned}
$$

But, from (20),

$$
e_{z_{\ell}}\left(t_{n}\right) e_{z_{\ell}}^{*}\left(t_{m}\right) \leftrightarrow e_{z_{k}}^{*}\left(t_{m}\right) \delta\left(z_{k} \boxminus z_{\ell}\right) .
$$

Since the transforms in both cases are the same, (21) follows. Interpreting

$$
e_{z_{k}}\left(\boxminus t_{m}\right)=e_{z_{k}}\left(0 \boxminus t_{m}\right),
$$

it follows from the basis exponential shift identity in (21) that

$$
e_{z_{k}}\left(\boxminus t_{m}\right)=e_{z_{k}}^{*}\left(t_{m}\right)
$$

- The TS-DFT of an inverted box minus shift is

$$
x^{*}\left(t_{m} \boxminus t_{n}\right) \leftrightarrow X^{*}\left(z_{k}\right) e_{z_{k}}^{*}\left(t_{m}\right)
$$

Proof:

$$
\begin{aligned}
x\left(t_{m} \boxminus t_{n}\right) & =\sum_{k=0}^{N-1} X\left(z_{k}\right) e_{z_{k}}\left(t_{m} \boxminus t_{n}\right) \partial z_{k} \\
& =\sum_{k=0}^{N-1}\left[X\left(z_{k}\right) e_{z_{k}}\left(t_{m}\right)\right] e_{z_{k}}^{*}\left(t_{n}\right) \partial z_{k}
\end{aligned}
$$

Conjugating both sides gives (23).

A special case of (23) is

$$
x^{*}\left(\boxminus t_{n}\right) \leftrightarrow X^{*}\left(z_{k}\right)
$$

Boxplus Operation. The box plus operation is defined as

$$
x\left(t_{m} \boxplus t_{n}\right):=x\left(t_{m} \boxminus\left(\boxminus t_{n}\right)\right) .
$$

\section{Boxplus Semigroup Property.}

$$
e_{z_{k}}\left(t_{n} \boxplus t_{m}\right)=e_{z_{k}}\left(t_{n}\right) e_{z_{k}}\left(t_{m}\right)
$$

Proof:

$$
\begin{aligned}
e_{z_{k}}\left(t_{n} \boxplus t_{m}\right) & =e_{z_{k}}\left(t_{n} \boxminus\left(\boxminus t_{m}\right)\right) \\
& =e_{z_{k}}\left(t_{n}\right) e_{z_{k}}^{*}\left(\boxminus t_{m}\right) \\
& =e_{z_{k}}\left(t_{n}\right) e_{z_{k}}\left(t_{m}\right) .
\end{aligned}
$$

Interpreting $e_{z_{k}}\left(\boxplus t_{m}\right)=e_{z_{k}}\left(0 \boxplus t_{m}\right)$, it follows that

$$
e_{z_{k}}\left(\boxplus t_{m}\right)=e_{z_{k}}\left(t_{m}\right) \text {. }
$$

Box Plus Commutivity. The commutative property of the box plus shift is

$$
x\left(t_{n} \boxplus t_{m}\right)=x\left(t_{m} \boxplus t_{n}\right) .
$$

Proof:

$$
x\left(t_{n} \boxplus t_{m}\right)=\sum_{k=0}^{N-1} X\left(z_{k}\right) e_{z_{k}}\left(t_{n} \boxplus t_{m}\right) \partial z_{k} .
$$

From (24),

$$
e_{z_{k}}\left(t_{n} \boxplus t_{m}\right)=e_{z_{k}}\left(t_{m} \boxplus t_{n}\right)
$$

From which (26) immediately follows.

- Box Plus TS-DFT. The TS-DFT of a box plus shift is

$$
x\left(t_{n} \boxplus t_{m}\right) \leftrightarrow X\left(z_{k}\right) e_{z_{k}}\left(t_{m}\right)
$$

Proof:

$$
\begin{aligned}
x\left(t_{n} \boxplus t_{m}\right) & =\sum_{k=0}^{N-1} X\left(z_{k}\right) e_{z_{k}}\left(t_{n} \boxplus t_{m}\right) \partial z_{k} \\
& =\sum_{k=0}^{N-1}\left[X\left(z_{k}\right) e_{z_{k}}\left(t_{m}\right)\right] e_{z_{k}}\left(t_{n}\right) \partial z_{k}
\end{aligned}
$$

from which (27) follows.

Box Plus Identity.

$$
x\left(\boxplus t_{n}\right)=x\left(t_{n}\right) .
$$


Proof: Using (25),

$$
\begin{aligned}
x\left(\boxplus t_{n}\right) & =\sum_{k=0}^{N-1} X\left(z_{k}\right) e_{z_{k}}\left(\boxplus t_{n}\right) \partial z_{k} \\
& =\sum_{k=0}^{N-1} X\left(z_{k}\right) e_{z_{k}}\left(t_{n}\right) \partial z_{k}=x\left(t_{n}\right) .
\end{aligned}
$$

\section{Time Scale Convolution and Correlation}

- Discrete time scale convolution between two functions is defined as

$$
x\left(t_{n}\right) * h\left(t_{n}\right):=\sum_{m=0}^{N-1} x\left(t_{m}\right) h\left(t_{n} \boxminus t_{m}\right) \partial t_{m} .
$$

- The TS-DFT of a convolution is the product of the transforms.

$$
x\left(t_{n}\right) * h\left(t_{n}\right) \leftrightarrow X\left(z_{k}\right) H\left(z_{k}\right) .
$$

Proof: Let $y=x * h$. Then

$$
\begin{aligned}
Y\left(z_{k}\right) & =\sum_{n=0}^{N-1} y\left(t_{n}\right) e_{z_{k}}^{*}\left(t_{n}\right) \partial t_{n} \\
& =\sum_{n=0}^{N-1}\left[\sum_{m=0}^{N-1} x\left(t_{m}\right) h\left(t_{n} \boxminus t_{m}\right) \partial t_{m}\right] e_{z_{k}}^{*}\left(t_{n}\right) \partial t_{n} \\
& =\sum_{m=0}^{N-1} x\left(t_{m}\right)\left[\sum_{n=0}^{N-1} h\left(t_{n} \boxminus t_{m}\right) e_{z_{k}}^{*}\left(t_{n}\right) \partial t_{n}\right] \partial t_{m} \\
& =\sum_{m=0}^{N-1} x\left(t_{m}\right)\left[H\left(z_{k}\right) e_{z_{k}}^{*}\left(t_{m}\right)\right] \partial t_{m} \\
& =\left[\sum_{m=0}^{N-1} x\left(t_{m}\right) e_{z_{k}}^{*}\left(t_{m}\right) \partial t_{m}\right] H\left(z_{k}\right)=X\left(z_{k}\right) H\left(z_{k}\right) .
\end{aligned}
$$

- Discrete time scale convolution is

- commutative

$$
x * h=h * x,
$$

- associative

$$
g *(h * x)=(g * h) * x,
$$

- and distributive over addition

$$
x *(g+h)=x * g+x * h .
$$

Proof: The proof follows immediately from the TS-DFT of a convolution in (29).

- Discrete time scale correlation between two functions is defined as

$$
x\left(t_{n}\right) \star h\left(t_{n}\right):=\sum_{m=0}^{N-1} x^{*}\left(t_{m}\right) h\left(t_{n} \boxplus t_{m}\right) \partial t_{m} .
$$

- Transformation of Correlation.

$$
x\left(t_{n}\right) \star h\left(t_{n}\right) \leftrightarrow X^{*}\left(z_{k}\right) H\left(z_{k}\right) .
$$

Proof:

$$
\begin{aligned}
x\left(t_{n}\right) \star h\left(t_{n}\right)= & \sum_{m=0}^{N-1} x^{*}\left(t_{m}\right) h\left(t_{n} \boxplus t_{m}\right) \partial t_{m} \\
\leftrightarrow & \sum_{n=0}^{N-1}\left[\sum_{m=0}^{N-1} x^{*}\left(t_{m}\right) h\left(t_{n} \boxplus t_{m}\right) \partial t_{m}\right] \\
& \times e_{z_{k}}^{*}\left(t_{n}\right) \partial t_{n} \\
= & \sum_{m=0}^{N-1} x^{*}\left(t_{m}\right) \\
& \times\left[\sum_{n=0}^{N-1} h\left(t_{n} \boxplus t_{m}\right) e_{z_{k}}^{*}\left(t_{n}\right) \partial t_{n}\right] \partial t_{m} \\
= & \sum_{m=0}^{N-1} x^{*}\left(t_{m}\right)\left[H\left(z_{k}\right) e_{z_{k}}\left(t_{m}\right)\right] \partial t_{m} \\
= & H\left(z_{k}\right)\left[\sum_{m=0}^{N-1} x\left(t_{m}\right) e_{z_{k}}^{*}\left(t_{m}\right) \partial t_{m}\right]^{*}
\end{aligned}
$$

from which (31) immediately follows.

- An alternate expression for correlation in (30) is

$$
x\left(t_{n}\right) \star h\left(t_{n}\right):=\sum_{m=0}^{N-1} x^{*}\left(t_{m} \boxminus t_{n}\right) h\left(t_{m}\right) \partial t_{m} .
$$

Proof: Using the power theorem

$$
\begin{aligned}
& \sum_{m=0}^{N-1} x^{*}\left(t_{n} \boxminus t_{m}\right) h\left(t_{n}\right) \partial t_{n} \\
= & \sum_{k=0}^{N-1}\left[X\left(z_{k}\right) e_{z_{k}}^{*}\left(t_{n}\right)\right]^{*} H\left(z_{k}\right) \partial z_{k} \\
= & \sum_{k=0}^{N-1}\left[X^{*}\left(z_{k}\right) H\left(z_{k}\right)\right] e_{z_{k}}\left(t_{n}\right) \partial z_{k}
\end{aligned}
$$

which is the same result as (31).

- Correlation obeys the following laws.

1) Reversal. If $y=x \star h$, and $\lambda=h \star x$, then

$$
\lambda\left(t_{n}\right)=y^{*}\left(\boxminus t_{n}\right) .
$$

2) Order

$$
\begin{aligned}
g \star(h \star x) & =h \star(g \star x) \\
& =(h * g) \star x .
\end{aligned}
$$

3) Distributive over addition

$$
x \star(g+h)=x \star g+x \star h .
$$

Proof:

1) Let $y\left(t_{n}\right)=x\left(t_{n}\right) \star h\left(t_{n}\right)$. Then

$$
y\left(t_{n}\right)=\sum_{k=0}^{N-1} X\left(z_{k}\right) H^{*}\left(z_{k}\right) e_{z_{k}}\left(t_{n}\right) \partial z_{k},
$$




\begin{tabular}{|c|c|c|c|c|}
\hline I. & $\begin{array}{l}\text { convolution } \\
\text { correlation }\end{array}$ & $\begin{array}{l}x * h \\
x \star h\end{array}$ & $\begin{array}{l}\leftrightarrow \\
\leftrightarrow\end{array}$ & $\begin{array}{l}X H \\
X^{*} H\end{array}$ \\
\hline II. & $\begin{array}{l}\text { commutative } \\
\text { associative } \\
\text { distributive }\end{array}$ & $\begin{array}{r}h * x \\
g *(h * x) \\
x *(g+h)\end{array}$ & $\begin{array}{l}= \\
= \\
=\end{array}$ & $\begin{array}{l}x * h \\
(g * h) * x \\
x * g+x * h\end{array}$ \\
\hline III. & $\begin{array}{l}\text { reversal } \\
\text { order } \\
\text { distributive }\end{array}$ & $\begin{array}{r}y\left(t_{n}\right)=x \star h \\
g \star(h \star x) \\
x \star(g+h)\end{array}$ & $\begin{array}{l}\rightarrow \\
= \\
= \\
=\end{array}$ & $\begin{array}{l}h \star x=y^{*}\left(\boxminus t_{n}\right) \\
h \star(g \star x) \\
(h * g) \star x \\
x \star g+x \star h\end{array}$ \\
\hline IV. & $\begin{array}{l}\text { correlation } \\
\text { convolution }\end{array}$ & $\begin{array}{l}x\left(t_{n}\right) \star h\left(t_{n}\right) \\
x\left(t_{n}\right) * h\left(t_{n}\right)\end{array}$ & $\begin{array}{l}= \\
=\end{array}$ & $\begin{array}{l}x^{*}\left(\boxminus t_{n}\right) * h\left(t_{n}\right) \\
x^{*}\left(\boxminus t_{n}\right) \star h\left(t_{n}\right)\end{array}$ \\
\hline
\end{tabular}

TABLE III

PROPERTIES OF CONVOLUTION AND CORRELATION. (I) TS-DFT. (II) CONVOlution. (III) CORRELATION. (IV) RELATIONSHIPS BETWEEN CONVOLUTION AND CORRELATION.

and

$$
y^{*}\left(t_{n}\right)=\sum_{k=0}^{N-1} X^{*}\left(z_{k}\right) H\left(z_{k}\right) e_{z_{k}}^{*}\left(t_{n}\right) \partial z_{k} .
$$

Thus

$$
\begin{aligned}
y^{*}\left(\boxminus t_{n}\right) & =\sum_{k=0}^{N-1} X\left(z_{k}\right) H^{*}\left(z_{k}\right) e_{z_{k}}^{*}\left(\boxminus t_{n}\right) \partial z_{k} \\
& =\sum_{k=0}^{N-1} H^{*}\left(z_{k}\right) X\left(z_{k}\right) e_{z_{k}}\left(t_{n}\right) \partial z_{k} . \\
& =h\left(t_{n}\right) \star x\left(t_{n}\right)=\lambda\left(t_{n}\right) .
\end{aligned}
$$

2) Follows immediately from

$$
g \star(h \star x) \leftrightarrow G^{*} H^{*} X .
$$

3) Follows immediately from the definition of correlation in (30).

- The relationship between convolution and correlation.

$$
x\left(t_{n}\right) \star h\left(t_{n}\right)=x^{*}\left(\boxminus t_{n}\right) * h\left(t_{n}\right)
$$

and

$$
\begin{aligned}
x\left(t_{n}\right) * h\left(t_{n}\right) & =x^{*}\left(\boxminus t_{n}\right) \star h\left(t_{n}\right) . \\
g \star(h \star x) & =h \star(g \star x) \\
& =(h * g) \star x .
\end{aligned}
$$

\section{FinAl REMARKS}

The TS-DFT establishes a generalization of the DFT to cases where points are not spaced uniformly. The generalization preserves properties of the conventional DFT, including derivative, shift, convolution and correlation relationships. The TS-DFT and its inverse are defined in (11) and (12). TS-DFT theorems are listed in Tables I, II and III.

Much work remains in the development of the foundations of the TS-DFT. The study of the mapping of time scales, $\mathbb{D}_{N}$, to frequency scales, $\mathbb{U}_{N}$, remains, for example, an open problem, as does filtering, sampling, and the mechanics of convolution and correlation [15].

\section{ACKNOWLEDGEMENT}

The authors are appreciative of John E. Miller's review and comments on this manuscript. This work was supported by NSF award CMMI-726996

\section{REFERENCES}

[1] Martin Bohner and Allan Peterson, Dynamic Equations on Time Scales: An Introduction with Applications, Birkhäuser, Boston (2001).

[2] P. Cheung and V. Kac, Quantum Calculus, Springer-Verlag, New York, 2002.

[3] J.J. DaCunha, Stability for time varying linear dynamic systems on time scales, J. Comput. Appl. Math. 176 (2005) 381-410.

[4] Davis, J.M., Henderson, J., Prasad, K.R., Yin, W.K.C.,"Solvability of a Nonlinear Second Order Conjugate Eigenvalue Problem on a Time Scale, Abs. Appl. Anal., 5 (2000) 91-99.

[5] Davis, J.M., J. Henderson, J., K.R. Prasad. Upper and Lower Bounds for the Solution of the General Matrix Riccati Differential Equation on a Time Scale, J. Comput. Appl. Math., 141 (2002) 133-145.

[6] John M. Davis, Ian A. Gravagne, Billy J. Jackson, Robert J. Marks II and Alice A. Ramos. The Laplace Transform on Time Scales Revisited, Journal of Mathematical Analysis Applications, 332 (2007) 1291-1307.

[7] J.M. Davis, I.A. Gravagne, B.J. Jackson, and R.J. Marks II, Controllability, observability, realizability, and stability of dynamic linear systems, Electronic Journal of Differential Equations 2009 (2009) 1-32.

[8] John M. Davis, Ian A. Gravagne and Robert J. Marks II. Convergence of Unilateral Laplace Transforms on Time Scales, Circuits, Systems, and Signal Processing (2010).

[9] John M. Davis, Ian A. Gravagne and Robert J. Marks II. Bilateral Laplace Transforms on Time Scales: Convergence, Convolution, and the Characterization of Stationary Stochastic Time Series, Circuits, Systems, and Signal Processing (2010).

[10] I.A. Gravagne, J.M. Davis, J.J. DaCunha, and R.J. Marks II, Bandwidth reduction for controller area networks using adaptive sampling, Proc. Int. Conf. Robotics and Automation, New Orleans, LA (2004) 5250-5255.

[11] S. Hilger, Ein Masskettenkalkül mit Anwendung auf Zentrumsmannigfaltigkeiten. Ph.D. thesis, Universität Würzburg, 1988.

[12] S. Hilger, Special Functions: Laplace and Fourier Transform on Measure Chains, Dynamic Systems and Applications 8 (1999) 471-488.

[13] R.J. Marks II, I.A. Gravagne, J.M. Davis, J.J. DaCunha. Nonregressivity in switched linear circuits and mechanical systems, Math. Comput. Modelling 43 (2006), 1383-1392.

[14] R.J. Marks II, I.A. Gravagne, and J.M. Davis, A generalized Fourier transform and convolution on time scales. J. Math. Anal. Appl. 340 (2008) 901-919.

[15] R.J. Marks II. Introduction to Shannon Sampling and Interpolation Theory. Springer- Verlag, New York, 1991.

[16] R.J. Marks II, Handbook of Fourier Analysis and Its Applications, Oxford University Press, (2009). HandbookOfFourierAnalysis.com .

$$
-\ltimes-
$$

